\title{
On the generic impossibility of truthful behavior: A simple approach $\star$
}

\author{
Carmen Beviá ${ }^{1}$ and Luis Corchón ${ }^{2}$ \\ 1 Departamento de Fundamentos, Universidad de Alicante, E-03071 Alicante, SPAIN \\ 2 Instituto Valenciano de Investigaciones Economicas and Departamento de Fundamentos, Universidad \\ de Alicante, E-03071 Alicante, SPAIN
}

Received: August 6, 1993; revised version November 24, 1993

Summary. We provide an elementary proof showing how in economies with an arbitrary number of agents an arbitrary number of public goods and utility functions quasi-linear in money, any efficient and individually rational mechanism is not strategy-proof for any economy satisfying a mild regularity requirement.

\section{Introduction}

From the seventies onwards, the study of strategy-proof mechanisms has been the subject of several papers. In most of these papers negative results are found. In voting theory, Gibbard [1] and Satterthwaite [6] prove that any strategy-proof mechanism is dictatorial. Hurwicz [2] shows that in two-goods, two-person, pure exchange economies, any efficient and individually rational allocation mechanism is manipulable (i.e. truth is not a dominant strategy for some agent) in some economy in the domain, provided that each agent has a positive initial endowment of at least one of the goods, and that a sufficiently wide class of convex preferences is covered (see Ledyard and Roberts [4] for the public good case). However, these results leave unanswered two questions: first, whether similar results are true under different assumptions on the set of admissible preferences and where there are more than two agents and/or more than two goods and second, how large is the set of economies for which truth is not a dominant strategy for some agent.

The work of Saijo [5] and Zhou ([7], [8]) addressed the first question. Saijo [5] studies the problem of the existence of strategy-proof and individually rational mechanisms when the Pareto efficient condition is obviated, and he proves that there is a non-constant mechanism that satisfies the above two requirements in economies with or without public goods. In the same paper Saijo proves that if the individually rational condition is strengthened, a new impossibility result appears.

\footnotetext{
* The authors wish to thank William Thomson, Salvador Barberá, José Angel Silva and an anonymous referee for helpful comments. The remaining errors are our exclusive responsibility. Financial support from DGICYT under project PB 91-0756 and the Instituto Valenciano de Investigaciones Económicas is gratefully acknowledged.
} 
More precisely, in public good economies, no strategy-proof mechanism yielding participative allocations exists. An allocation is participative if every participant's bundle is no worse than the best bundle that can be achieved solely by the participant's endowment and technology, without using that of other participants. On the other hand, new proof techniques based on the identification of the geometric properties of the Pareto efficient set, enable Zhou [7] to prove fresh results. He shows that in the domain of pure exchange economies with two agents and arbitrarily many goods in which both agents' utility functions are continuous, strictly concave and increasing, any efficient and non-inversely-dictatorial allocation mechanism is manipulable for some economy in the domain. In economies with pure public goods and with continuous and convex preferences, the same author [8] proves that any strategy-proof mechanism is dictatorial whenever the range of the mechanism has, at least, dimension two.

Hurwicz and Walker [3] provide an answer to the second question. Using advanced techniques, they proved that, in economies with quasi-linear utility functions any strategy-proof mechanism defined on a convex and semi-open set of strictly concave and continuous valuations of the public goods, will not yield Pareto efficient allocations on any open and dense set of preference profiles, except by producing allocations that lie on the relative boundary of the feasible set. (Notice that a mechanism that gives the total endowment to participant one regardless of the preference announcements of other participants is strategy-proof and Pareto efficient). This result shows that, with the exception of constant mechanisms, under some restrictions on the set of admissible preferences, Pareto efficiency and strategy-proofness are two generally incompatible requirements.

In this paper we prove a dual result to that of Hurwicz and Walker [3], in the following sense: they assume that the mechanism is strategy-proof and prove that efficiency is violated almost everywhere and we assume that the mechanism yields efficient and individual rational allocations and we prove that non-manipulability is violated almost everywhere. In other words, we prove that in economies with an arbitrary number of agents, an arbitrary number of public goods and quasi-linear utility functions, any efficient and individually rational mechanism is manipulable for any regular economy i.e., an economy where the valuations of the public goods are strictly concave, $C^{2}$ and their Gaussian curvature are non-vanishing (see Theorem 1 below). This result differs from that of Hurwicz and Walker [3] in the assumptions - we require individual rationality, but we do not assume continuity of the mechanism - and that we use only elementary techniques. Furthermore, our Theorem 1 identifies those economies (the regular ones) for which to announce the truth is not a dominant strategy for some agent. Hurwicz and Walker did not, since they only obtain a generic result based on the topology they used (we do not know if their result is true for other topologies. Also, as it is exemplified by the classical example of the rational and real numbers, an open and dense set is not necessarily very big). Moreover, our approach allows for a graphical representation when there are two agents and one public good. Finally, we show that our result remains true without using linear economies, that is, restricting the domain of the mechanism to the regular economies. It goes without saying that it is very easy to adapt our argument and to show that in exchange economies with an arbitrary number of 
agents, an arbitrary number of private goods and quasi-linear utility functions, any efficient and individually rational mechanism is manipulable for any regular economy (appropriately defined).

The rest of this note goes as follows. The next Section explains the model and the main definitions and Section 3 gathers our main results.

\section{The model and definitions}

There are $n$ agents in the society. There is one private good (it is sometimes helpful to think of this good as "money"), and there are $m$ public goods. Public goods are produced from the private good by means of a constant returns to scale technology represented by a linear cost function $c($.). A consumption for agent $i$ is a pair $\left(x_{i}, y\right) \in \mathbb{R}_{+}^{1+m}$ where $x_{i} \in \mathbb{R}_{+}$is the private good he consumes on his own, and $y \in \mathbb{R}_{+}^{m}$ is the vector of public goods. Let $\left(w_{i}, 0\right) \in \mathbb{R}_{+}^{1+m}$ be agent $i$ 's initial endowment (i.e., we assume that there are initially no public goods). Let

$$
X=\left\{(x, y) \in \mathbb{R}_{+}^{n+m} / \sum_{i=1}^{n}\left(w_{i}-x_{i}\right)=c(y)\right\}
$$

be the set of feasible allocations. Each agent $i$ has a preference relation defined over $\mathbb{R}_{+}^{1+m}$ represented by a quasi-linear (on money) utility function, that is, $u_{i}: \mathbb{R}_{+}^{1+m} \rightarrow \mathbb{R}$ such that $u_{i}\left(x_{i}, y\right)=v_{i}(y)+x_{i}$. Let $U_{i}$ be the (exogenously given) set of admissible preferences for agent $i$. $U$ denotes the product space $U=U_{1} \times \cdots \times U_{n}$. A generic point $u=\left(u_{1}, \ldots, u_{n}\right)$ in $U$ is called a preference profile. Sometimes we will refer to $u \in U$ as an economy, and this will be written as $\left(u_{i}, u_{-i}\right)$, where $u_{-i}=\left(u_{1}, \ldots, u_{i-1}\right.$, $\left.u_{i+1}, \ldots, u_{n}\right)$.

An economy $u \in U$ is said to be regular if for all $i=1, \ldots, n, v_{i}($.$) is C^{2}$ and strictly concave with non-vanishing Gaussian curvature ${ }^{1}$.

Let us denote by $U^{R}$ the space of all regular economies. Let $U^{L}$ be the space of all linear economies.

Given an economy $u \in U$, a feasible allocation $(x, y)$ is Pareto efficient for $\mathbf{u}$ if no other feasible allocation $\left(x^{\prime}, y^{\prime}\right)$ exists such that $u_{i}\left(x_{i}^{\prime}, y^{\prime}\right) \geq u_{i}\left(x_{i}, y\right)$ for all $i$ with strict inequality for at least one agent. Notice that the efficient vector of public goods is unique for any $u$ since preferences are quasi-linear.

Given an economy $u \in U$, a feasible allocation $(x, y)$ is individually rational for $\mathbf{u}$ if $u_{i}\left(x_{i}, y\right) \geq u_{i}\left(w_{i}, 0\right)$ for all $i$.

An allocation $\left(x^{L}, y^{L}\right)$ is a Lindahl allocation for $\mathbf{u} \in \mathbf{U}$, if it is feasible and there is a price vector $p_{i} \in \mathbb{R}_{+}^{m}$ one for each $i$ such that,

(1) $x_{i}^{L}+p_{i} y^{L} \leq w_{i}, \quad i=1, \ldots, n$

(2) $u_{i}\left(x_{i}, y\right)>u_{i}\left(x_{i}^{L}, y^{L}\right)$ implies $x_{i}+p_{i} y>w_{i}, \quad i=1, \ldots, n$

(3) $\sum_{i=1}^{n} p_{i} y^{L}-c\left(y^{L}\right) \geq \sum_{i=1}^{n} p_{i} y-c(y) \forall y \in \mathbb{R}_{+}^{m}$

An allocation $\left(x^{M}, y^{M}\right)$ is called a monopoly point for $\mathbf{u} \in \mathbf{U}$ when agent $\mathbf{i}$ behaves

\footnotetext{
${ }^{1}$ Our results remains true if we redefine a regular economy as one such that $v($.$) is concave and C^{2}$ for all agents and it has a non-vanishing Gaussian curvature for at least two agents.
} 
as a monopolist if it is feasible and there is a price vector $p_{j} \in \mathbb{R}_{+}^{m}$ one for each $j$ such that

(1') $x_{j}^{M}+p_{j} y^{M} \leq w_{j}, \quad j=1, \ldots, n$

(2) $u_{j}\left(x_{j}, y\right)>u_{j}\left(x_{j}^{M}, y^{M}\right)$ implies $x_{j}+p_{j} y>w_{j}$ for all $j \neq i$

(3) $\sum_{j=1}^{n} p_{j} y^{M}-c\left(y^{M}\right) \geq \sum_{j=1}^{n} p_{j} y-c(y) \forall y \in \mathbb{R}_{+}^{m}$

(4') $u_{i}\left(x_{i}^{M}, y^{M}\right) \geq u_{i}\left(x_{i}, y\right)$ for all $(x, y) \in X$ satisfying $\left(1^{\prime}\right),\left(2^{\prime}\right)$ and $\left(3^{\prime}\right)$.

Since the cost function is convex, condition (3) and $\left(3^{\prime}\right)$ can be replaced by $\sum_{i=1}^{n} p_{i k}=\frac{\partial c(y)}{\partial y_{k}}, \quad k=1, \ldots, m$.

It is clear that for any regular economy, both the Lindahl allocation and the monopoly point exist.

A (direct) mechanism is a function $f: U \rightarrow X$, which maps each preference profile into the set of feasible allocations. Let $f_{i}(u)=\left(x_{i}, y\right)$ be the consumption obtained by $i$ if the profile $u$ is announced.

A mechanism $f$ is efficient if for any $u \in U, f(u)$ is a Pareto efficient allocation for $u$.

A mechanism $f$ is individually rational if for any $u \in U, f(u)$ is an individually rational allocation for $u$.

A mechanism $f$ is strategy-proof if for any agent $i$, any $u \in U$ and any $u_{i}^{\prime} \in U_{i}$, $u_{i}\left(f_{i}\left(u_{i}, u_{-i}\right)\right) \geq u_{i}\left(f_{i}\left(u_{i}^{\prime}, u_{-i}\right)\right)$.

A mechanism $f$ is non-manipulable at profile $u$ if for any agent $i$, and any $u_{i}^{\prime} \in U_{i}$, $u_{i}\left(f_{i}\left(u_{i}, u_{-i}\right)\right) \geq u_{i}\left(f_{i}\left(u_{i}^{\prime}, u_{-i}\right)\right)$.

Notice that the latter of these two definitions is weaker than the former and is the one which will be used in Theorem 1 below. Thus, our results are stronger, for example, than the one obtained by Ledyard and Roberts [4], because they use the former definition.

\section{The main result}

We start this section proving that given a regular economy, the agent who behaves as a monopolist is strictly better off in the monopoly allocation than in the Lindahl allocation (see Lemma 1 below). This Lemma will be used to prove our main result (Theorem 1).

Lemma 1: Given a regular economy $u \in U$, if $\left(x_{i}^{M}, y^{M}\right) \gg 0$ and $\left(x_{i}^{L}, y^{L}\right) \gg 0$, then $u_{i}\left(x_{i}^{L}, y^{L}\right)<u_{i}\left(x_{i}^{M}, y^{M}\right)^{2}$.

Proof. Since $\left(x^{L}, y^{L}\right)$ is the Lindahl allocation for $u, y^{L}$ satisfies:

$$
\sum_{i=1}^{n} \frac{\partial v_{i}(y)}{\partial y_{j}}=\frac{\partial c(y)}{\partial y_{j}}, j=1, \ldots, m
$$

if $\left(x^{M}, y^{M}\right)$ is the allocation obtained if agent $i$ behaves as a monopolist, we obtain that,

\footnotetext{
${ }^{2}$ Vector inequalities $\gg,>, \geq$.
} 


$$
p_{i k}=\frac{\partial c(y)}{\partial y_{k}}-\sum_{1 \neq i} \frac{\partial v_{1}(y)}{\partial y_{k}}, \quad k=1, \ldots, m
$$

Then, by the program that agent $i$ solves and because of the linearity of the cost function, for all $k=1, \ldots, m$,

$$
\frac{\partial v_{i}(y)}{\partial y_{j}}-\frac{\partial c(y)}{\partial y_{j}}+\sum_{1 \neq i} \frac{\partial v_{1}(y)}{\partial y_{j}}+\sum_{1 \neq i} \sum_{k=1}^{m} \frac{\partial^{2} v_{1}(y)}{\partial y_{k} \partial y_{j}} y_{k}=0
$$

Therefore,

$$
\sum_{i=1}^{n} \frac{\partial v_{i}(y)}{\partial y_{j}}=\frac{\partial c(y)}{\partial y_{j}}-\sum_{1 \neq i} \sum_{k=1}^{m} \frac{\partial^{2} v_{1}(y)}{\partial y_{k} \partial y_{j}} y_{k}, \quad j=1, \ldots, m
$$

Since $\left(x^{L}, y^{L}\right)$ satisfies $\left(1^{\prime}\right),\left(2^{\prime}\right),\left(3^{\prime}\right), u_{i}\left(x_{i}^{L}, y^{L}\right) \leq u_{i}\left(x_{i}^{M}, y^{M}\right)$, then it is enough to prove that $u_{i}\left(x_{i}^{L}, y^{L}\right) \neq u_{i}\left(x_{i}^{M}, y^{M}\right)$. Suppose that $u_{i}\left(x_{i}^{L}, y^{L}\right)=u_{i}\left(x_{i}^{M}, y^{M}\right)$. Then $y^{L}$ must satisfy [2]. Since $y^{L}$ satisfies [1], this implies that

$$
\sum_{1 \neq i} \sum_{k=1}^{m} \frac{\partial^{2} v_{1}\left(y^{L}\right)}{\partial y_{k} \partial y_{j}} y_{k}^{L}=0, \quad j=1, \ldots, m
$$

Let $v(y)=v_{1}(y)+\cdots+v_{i-1}+v_{i+1}+\cdots+v_{n}(y)$. Since $v_{i}$ is strictly concave for all $i$, $v$ is strictly concave. Then we can write [3] as $H v\left(y^{L}\right) y^{L}=0$, where $H v($.$) is the Hessian$ matrix of the function $v$. Then $\left(y^{L}\right)^{t} H v\left(y^{L}\right) y^{L}=0$, but this is a contradiction since $y^{L} \neq 0$ and $\left(y^{L}\right)^{t} H v\left(y^{L}\right) y^{L}<0$ because $v$ is strictly concave with a non-vanishing Gaussian curvature.

Now we are prepared to prove our main result

Theorem 1. Let $f: U \rightarrow X$ with $f(u) \gg 0 \forall u \in U$ be an efficient and individually rational mechanism with $U^{L} \subseteq U$. Then, $f$ is manipulable at any regular economy in $U$ such that $\left(x_{i}^{M}, y^{M}\right) \geqq 0$ and $\left(x_{i}^{L}, y^{L}\right) \gg 0$.

Proof. We first consider the case of $n=2, m=1$, in order to offer a graphical insight on how the proof works. A formal proof is then offered.

Given a regular economy $u \in U$ (see Figure 1 ) let $B-B^{\prime}$ be the set of all efficient and individually rational allocations for $u$. Since mechanism $f$ is efficient and individually rational, $f(u)$ must be at some point between $B$ and $B^{\prime}$. Suppose that it is between $B$ and $L$ ( $L$ is the Lindahl allocation for $u$ ). Then agent 1 can misrepresent his utility function by sending a constant marginal rate of substitution. equal to his monopoly prices (the dotted line in Figure 1). He obtains an allocation in the new efficient and individually rational set $M-F$ ( $M$ is his monopoly point). For any point therein, agent 1 is better off than before. If $f(u)$ is at some point between $L$ and $B^{\prime}$, agent 2 would manipulate accordingly.

We now provide a formal proof of the Theorem. Let $u \in U$ be a regular economy such satisfying the properties in the theorem, and let $(x, y)=f\left(u_{i}, u_{-i}\right)$. Since $\left(x^{L}, y^{L}\right)$ and $(x, y)$ are Pareto efficient, there exists $i$ such that

$$
x_{i}+v_{i}(y) \leq x_{i}^{L}+v_{i}\left(y^{L}\right) .
$$

where $x_{i}+v_{i}(y) i=1, \ldots, n$ are the true utility functions relative to the economy $u$. 
Suppose that agent $i$ sends a utility function

$$
u_{i}^{\prime}\left(x_{i}, y\right)=\sum_{k=1}^{m} p_{i k}^{M} y_{k}+x_{i}
$$

where $p_{k i}^{M}$ are the monopoly prices of the economy $u$ with $i$ as a monopolist. Let $\left(x^{\prime}, y^{\prime}\right)=f\left(u_{i}^{\prime}, u_{-i}\right)$, and let $\left(x_{i}^{M}, y^{M}\right)$ be the consumption of agent $i$ in the economy $u$ when he is a monopolist. We claim that

$$
x_{i}^{M}+v_{i}\left(y^{M}\right) \leq x_{1}^{\prime}+v_{i}\left(y^{\prime}\right) .
$$

Suppose that

$$
x_{i}^{M}+v_{i}\left(y^{M}\right)>x_{i}^{\prime}+v_{i}\left(y^{\prime}\right) .
$$

Since $\left(x^{M}, y^{M}\right)$ and $\left(x^{\prime}, y^{\prime}\right)$ are Pareto efficient allocations for the economy $\left(u_{i}^{\prime}, u_{-i}\right)$, our assumptions imply that $y^{M}=y^{\prime}$, so $x_{i}^{M}>x_{i}^{\prime}$. At the monopoly point the budget constraint for $i$ is satisfied, so $w_{i}=\sum_{k=1}^{m} p_{k i}^{M} y_{k}^{M}+x_{i}^{M}>\sum_{k=1}^{m} p_{k i}^{M} y_{k}^{\prime}+x_{i}^{\prime}$, which is a contradiction because the mechanism is individually rational. This proves the claim. Therefore, and by the previous lemma

$$
x_{i}+v_{i}(y) \leq x_{i}^{L}+v_{i}\left(y^{L}\right)<x_{i}^{M}+v_{i}\left(y^{M}\right) \leq x_{i}^{\prime}+v_{i}\left(y^{\prime}\right)
$$

which shows that mechanism $f$ is manipulable at $u$.

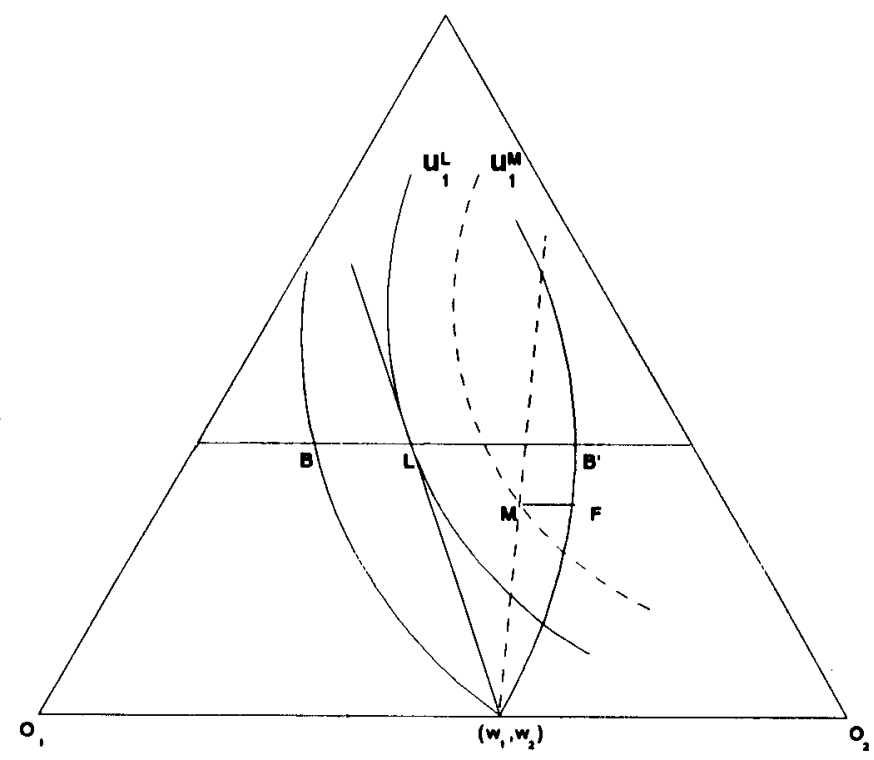

Figure 1.

In Theorem 1 we have proved the impossibility of truthful behavior on the set of regular economies when efficiency and individual rationality are imposed on the mechanism. It is easy to show that the set of strictly concave functions with nonvanishing Gaussian curvature is dense, with the Punctual Topology, in the set of 
concave functions. Thus, the set of regular economies is dense in the set of admissible preferences. Therefore an individually rational and Pareto efficient mechanism is manipulable almost everywhere.

An examination of the proof of Theorem 1 shows that whenever a regular economy is considered, an agent has incentives to deviate announcing an appropriate linear utility function. By the above observation about the density of the regular economies, it is possible to carry out the proof by taking only strictly concave (with non-vanishing Gaussian curvature) valuations of the public goods, i.e. Theorem 1 remains true if the set of admissible preferences is restricted to be the set of regular economies.

\section{References}

1. Gibbard, A.: Manipulation of voting schemes: a general result. Econometrica 41, 587-602 (1973)

2. Hurwicz, L.: On informationally decentralized systems. In: C.Mc Guire and R. Radner (eds.) Decision and organization. Amsterdam: North Holland 1972

3. Hurwicz, L., Walker, M.: On the generic non-optimality of dominant strategy allocation mechanisms. Econometrica 58, 683-704 (1990)

4. Ledyard, J., Roberts, J. D.: On the incentive problem with public goods. D.P., 116, Northwestern University 1974

5. Saijo, T.: Incentive compatibility and participativeness in public good economies. Institute of Socio-Economic Planning, University of Tsukuba 1990

6. Satterthwaite, M. A.: Strategy proofness and Arrow's conditions: existence and correspondence theorems for voting procedures and social welfare functions. J. Econ. Theory 10, 187-217 (1975)

7. Zhou, L.: Inefficiency of strategy-proof allocation mechanisms in pure exchange economies. Soc. Choice Welfare 8, 247-254 (1991)

8. Zhou, L.: Impossibility of strategy-proof mechanisms for economies with pure public goods. Rev. Econ. Stud. 58, 107-119 (1990) 